\title{
Reduced Basis Approximations for Maxwell's Equations in Dispersive Media
}

\section{Hess ${ }^{1}$, J. Hesthaven², P. Benner ${ }^{1}$}

${ }^{1}$ Max Planck Institute for Dynamics of Complex Technical Systems, Computational Methods in Systems and Control Theory, Magdeburg, Germany

'Ecole Polytechnique Fédérale de Lausanne, Switzerland

\section{Motivation}

Simulation of electromagnetic and optical wave propagation in, e.g. water, fog or dielectric waveguides requires modeling of linear, temporally dispersive media. Using a POD-greedy sampling driven by an error indicator, we seek to generate a reduced model which accurately captures the dynamics. Typically, the reduced basis model reduction reduces the model order by a factor of more than 100 , while maintaining an approximation error of less than $1 \%,[1,2]$

\section{Simulation}

The time-harmonic Maxwell's equations in the electric field $E$ are coupled with the polarization $P$

$$
\begin{aligned}
\frac{1}{\mu_{0}} \nabla \times \nabla \times E+\epsilon_{0} \epsilon_{\infty} \partial_{t}^{2} E & =f-\partial_{t}^{2} P, \\
\partial_{t} P+\frac{1}{\tau} P & =\frac{\epsilon_{0}\left(\epsilon_{s}-\epsilon_{\infty}\right)}{\tau} E,
\end{aligned}
$$

which is derived from adding relaxation polarization to the instantaneous polarization $\epsilon_{0} \epsilon_{r} E(t, \mathbf{x})$, namely

$$
\epsilon_{0} \epsilon_{\infty} E(t, \mathbf{x})+\epsilon_{0} \int_{-\infty}^{t} E(t-\tau, \mathbf{x}) \chi(\tau) d \tau
$$

This is called a Debye relaxation and models polar molecules with a permanent dipole moment [3]. Under the application of an external field, the molecules rotate and cause friction, which leads to an exponential damping.

Material parameters:

- relative permittivity in the static frequency limit $\epsilon_{\mathrm{s}}$

- relative permittivity in the infinite frequency limit $\epsilon_{\infty}$

- relaxation time $\tau$

- vacuum permittivity $\epsilon_{0}$ and vacuum permeability $\mu_{0}$

Typical material values are for instance for water $\epsilon_{\infty}=1.80, \epsilon_{s}=81.00, \tau=9.400 \times 10^{-12} \mathrm{~s}$ Simulation details

- unit square with zero Dirichlet boundary conditions (PEC - perfectly electric conducting)

- broadband input source $f$ modeled as a Gaussian pulse

- computational mesh has $9^{\prime} 680$ degrees of freedom Nédélec edge elements

- Runge-Kutta-Nyström time-stepping in the electric field

- explicit Euler in the polarization

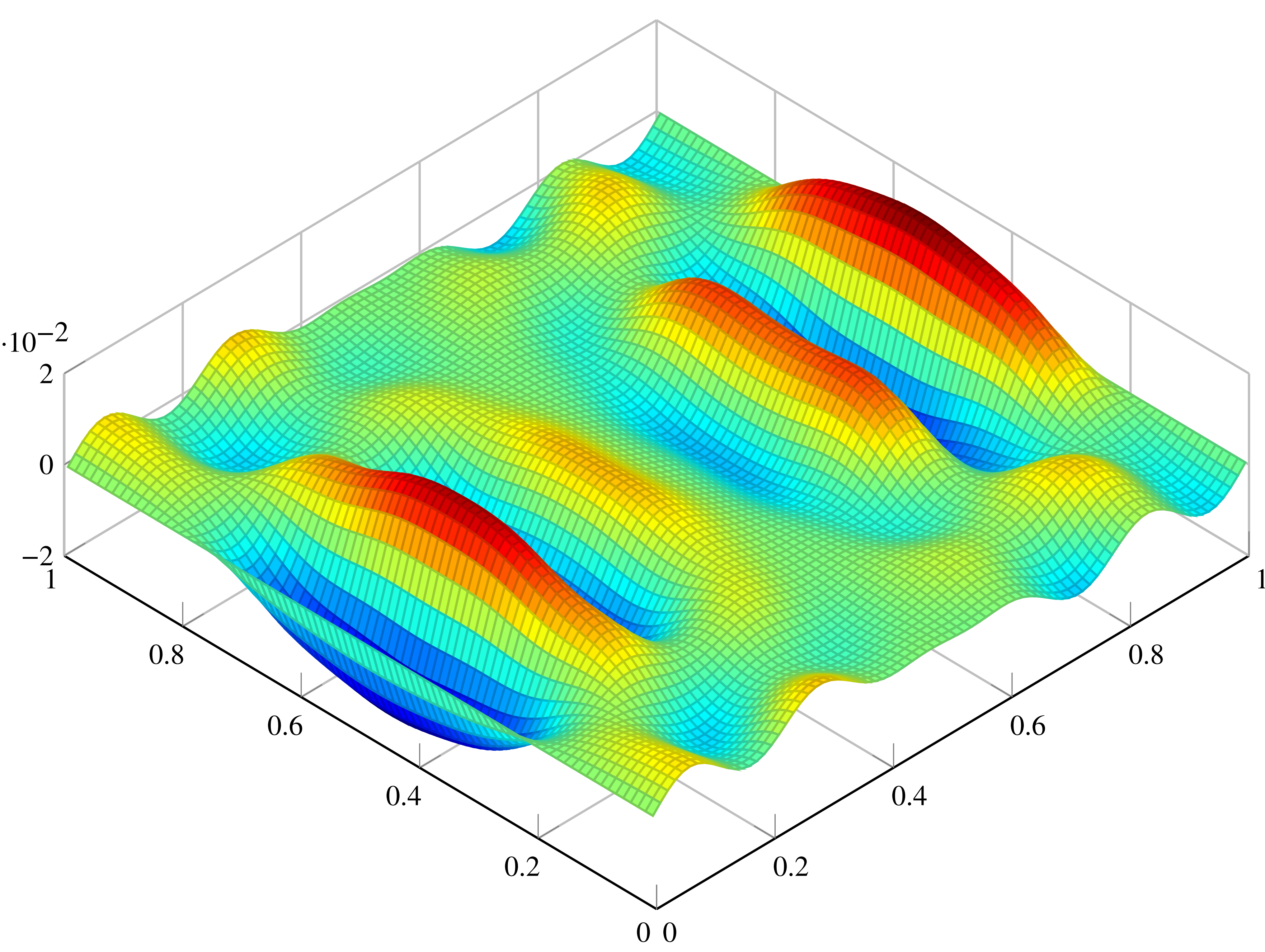

Wave propagation of one component of the electric field after normalization.

\section{References}

1] G. Rozza, D.B.P. Huynh and A.T. Patera, Reduced Basis Approximation and A Posteriori Error Estimation for Affinely Parametrized Elliptic Coercive Partial Differential Equations, Arch. Comput. Estimation for Affinely Parametrized Elli

[2] M. Hess and P. Benner, Fast Evaluation of Time-Harmonic Maxwell's Equations Using the Re duced Basis Method, IEEE Transactions on Microwave Theory and Techniques, 61(6), pp 22652274,2013

[3] K. E. Oughstun, Electromagnetic and Optical Pulse Propagation 1: Spectral Representations in Temporally Dispersive Media, Electromagnetic and Optical Pulse Propagation. Springer, 2006. [4] H. Cheng, Z. Gimbutas, P. G. Martinsson and V. Rokhlin, On the Compression of Low Rank Matrices, SIAM J. Sci. Comp. 26(4), pp. 1389-1404, 2005.

[5] Y. Zhang, L. Feng, S. Li and P. Benner, Accelerating PDE constrained optimization by the reduced basis method: application to batch chromatography, International Journal for Numerica Methods in Engineering, 2015.

\section{Parametric Model Order Reduction}

The model is parametrized in $\tau$ and $\Delta_{\epsilon}=\epsilon_{s}-\epsilon_{\infty}$, defining the 2-dimensional parameter domain $D$. We seek to generate a reduced model which accurately captures the dynamics in the parameter domain $D$ over timescales of a few nanoseconds.. A computationally intensive offline phase determines the projection space $V_{N}$, the parameter-independent parts of the affine form and the quantities required for error estimation. The online phase solves the reduced order PDE and evaluates the error estimator independently of the originally large system dimension.

It holds

$$
\Delta(\nu)=\left\|E(\cdot, \nu)-E_{N}(\cdot, \nu)\right\| \leq \sum_{k=1}^{n} a^{k} b_{n-k},
$$

where

$$
a=\sup _{n \in\left\{1, \ldots, n_{T}\right\}} \sup _{w \neq y} \frac{\|w-y\|}{\left\|f\left(w, t_{n} ; \nu\right)-f\left(y, t_{n} ; \nu\right)\right\|},
$$

is the inverse Lipschitz constant and $b_{n}$ is the residual at timestep $n$. Rigorous error estimation requires an upper bound on the inverse Lipschitz constant, since this is extremely costly, we use $a=1$ here

\section{POD-Greedy Algorithm}

- sampled parameter domain $D$ in $\tau$ and $\Delta_{\epsilon}=\epsilon_{\infty}-\epsilon_{s}$

- solve model at parameter configuration $\nu_{1}$ for $E\left(t_{i} ; \nu_{1}\right)$ and $P\left(t_{i} ; \nu_{1}\right)$, where $i=1, \ldots, n_{T}$ and set solve
$k=1$

- POD of trajectory $\Rightarrow$ projection basis $V_{N}$

- while $\max \equiv \Delta(\nu)>$ tolerance

- $\quad$ set $\mathrm{k}=\mathrm{k}+1$;

- set $\nu_{k}=\arg \max \equiv \Delta(\nu)$

- $\quad$ solve model for trajectory $E\left(\cdot ; \nu_{k}\right)$ and $P\left(\cdot ; \nu_{k}\right)$

- $\quad$ POD of trajectory $\Rightarrow$ append modes to $V_{N}$

- end while

\section{Interpolatory Decomposition}

Since the singular value decomposition (SVD) in the POD step is costly, an interpolatory decomposition (ID) is considered as an alternative, [3]. The potential advantage is, that computation times are lower than for an SVD. On the other hand, the interpolatory decomposition does not generate orthonormal matrices. This model also includes the adaptive snapshot selection [5] to enhance the orthonormal matrices. This model also includes the adaptive
offline times by compressing the trajectories before the SVD.

The interpolatory decomposition of a matrix $A \in \mathbb{R}^{m \times n}$ is a decomposition into $U \in \mathbb{R}^{m \times k}, B \in \mathbb{R}^{k \times k}$ and $V \in \mathbb{R}^{m \times k}$ as

$$
A \approx U \circ B \circ V^{T}
$$

where $B$ is a $k \times k$ submatrix of $A$. It expresses each of the columns of $A$ as a linear combination of $k$ selected columns of $A$ and analogously for the rows. This selection defines the $k \times k$ submatrix $B$ of $A$, and in the resulting system of coordinates, the action of $A$ is represented by the action of its submatrix $B$.

\section{Numerical Results}

\begin{tabular}{c|c|c|c} 
Method & 500 timesteps & 1500 timesteps & 3000 timesteps \\
\hline SVD & $2.9 \mathrm{~s}$ & $10.9 \mathrm{~s}$ & $26.6 \mathrm{~s}$ \\
ID & $0.9 \mathrm{~s}$ & $4.4 \mathrm{~s}$ & $13.2 \mathrm{~s}$
\end{tabular}

Comparison of SVD and ID computation times for different number of timesteps.

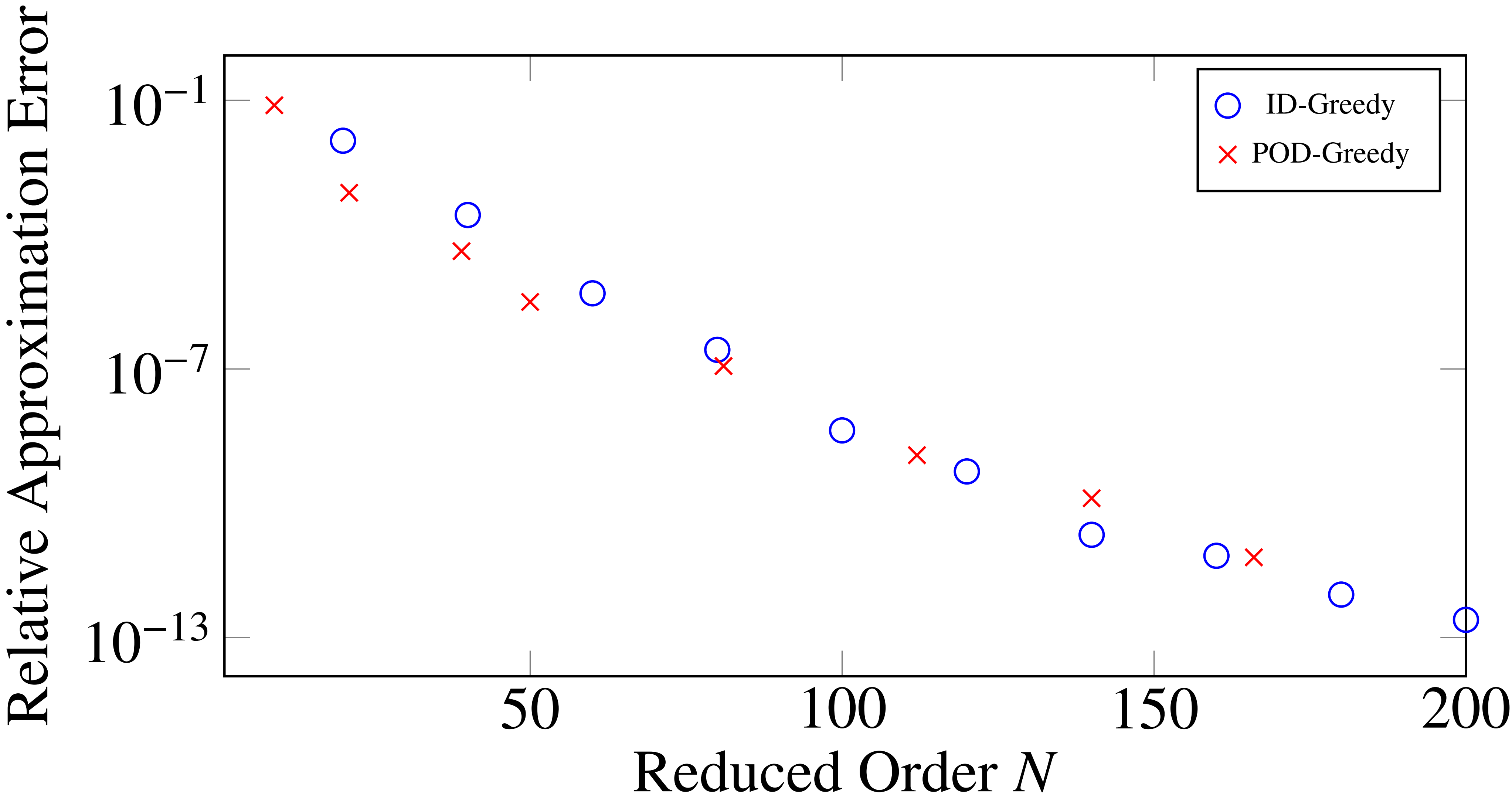

Comparison of SVD (red) and ID (blue) generated models. Shown is the maximum relative error in the electric field over the sampled parameter domain. 